\section{Christoph Bachmann}

\section{Die Situation der Schweizer Apotheken}

In der Schweiz gibt es etwa 1700 Apotheken (2014: 1764 [1]), die ungefähr gleichmässig auf die Deutschschweiz sowie die Westschweiz und den Kanton Tessin verteilt sind. Die beiden Regionen haben ungefähr gleich viele Apotheken, obwohl die deutsche Schweiz etwa 70\% der Schweizer Bevölkerung ausmacht, weil in vielen Deutschschweizer Kantonen die Ärzte das Recht haben, Medikamente in ihrer Praxis direkt zu verkaufen. Darum ist die Apothekendichte in der Deutschschweiz viel geringer als in der Romandie und dem Tessin.

Dafür ist in der Schweiz der Internethandel mit Medikamenten verboten, und der Anteil der OTC-Medikamente am Gesamtumsatz ist in Schweizer Apotheken viel grösser als z.B. in deutschen Apotheken, die nur eine geringe Anzahl an OTC-Medikamenten verkaufen, weil dort ein Grossteil dieser Medikamentengruppe per Internethandel vertrieben wird.

Wie in allen Ländern gibt es auch in der Schweiz ganz unterschiedliche Apotheken: Von der kleinen Quartierapotheke mit einem nur begrenzten Sortiment an Nichtmedikamenten bis zur sehr grossen Apotheke in Einkaufszentren oder im Bahnhofshopping gibt es jede Art von Grösse. Von den 1764 Apotheken gehören etwa ein Drittel zu Apotheken-Ketten wie Amavita, SUN STORE oder BENU usw. [2], in denen die verantwortliche Apo-

\title{
Obstipationsberatung in der Apotheke mit einem tibetischen Vielstoffgemisch: Umsetzung wissenschaftlicher Fakten in Beratungsgesprächen
}

In einer Apotheke werden täglich Laxativa verkauft. In vielen Fällen findet dies in Form eines nur einige Augenblicke dauernden Verkaufsgesprächs statt. Es kommt aber auch recht oft vor, dass eine Person von der Fachkompetenz eines Apothekers profitiert, um sich umfassend beraten zu lassen. Je nach Ausrichtung der betreffenden Apotheke wird in einem solchen Fall oft ein pflanzliches Vielstoffgemisch aus der Tibetischen Medizin empfohlen, das einen Multi-Target-Wirkungsmechanismus hat.

thekerin bzw. der verantwortliche Apotheker Geschäftsführer, nicht aber Eigentümer der betreffenden Apotheke ist. Die restlichen Apotheken sind unabhängig, schliessen sich aber immer mehr zu sogenannten Gruppierungen zusammen, um überleben $\mathrm{zu}$ können.

\section{Die Beratung in der Apotheke}

Es gibt in der Schweiz sicher auch Apotheken-Ketten, in denen die Kundschaft ausführlich beraten wird. Wegen des zentralen Einkaufs und Marketings von Apotheken-Ketten bleibt die Beratungsfreiheit der entsprechenden Apothekerinnen und Apotheker aber beschränkt. Und es gibt natürlich auch unabhängige Apotheken, in denen der Verkauf von möglichst vielen Präparaten das oberste Prinzip ist, worunter die Beratung leidet.

Es gibt in der letzten Zeit wieder zunehmend mehr Apotheken mit einer komplementärmedizinischen Ausrichtung und Beratung. Leider haben die Schweizer Apotheken, wahrscheinlich aus Profilierungsgründen gegenüber der Ärzteschaft, während vielen Jah- ren das Gebiet der Komplementärmedizin vernachlässigt und dieses weitgehend den Drogerien überlassen. Diese haben sich in der Folge als Kompetenzzentren für Komplementärmedizin verstanden. Nur allmählich begannen wieder vermehrt Apotheken, sich auf die Kompetenz der pharmazeutischen Biologie bzw. der Pharmakognosie zu besinnen und das Potenzial der Natur wieder medizinisch zu nutzen. Dies geschah sicher auch im Zusammenhang mit dem Beginn der klinischen Forschung in der Phytotherapie, deren Anfänge in den 1980erJahren liegen. Viele Apotheken versuchen heute, komplementärmedizinische Beratung mit medizinischwissenschaftlichen Kenntnissen $\mathrm{zu}$ verknüpfen und die Literatur über natürliche Therapien und Behandlungen kritisch zu sichten, um innerhalb der Komplementärmedizin das empfehlen und einsetzen zu können, was sich auf einer wissenschaftlichen Grundlage bewegt oder was wenigstens als wissenschaftlich einigermassen möglich erscheint. Damit grenzen sie sich von vielen Vertretern der Komplementärmedizin ab, die alles, was natürlich ist, als gut und wirksam bezeichnen und

\section{KARGER}

(C) 2016 S. Karger GmbH, Freiburg

Fax +497614520714 
Tab. 1. Zusammensetzung und Dosis der Inhaltsstoffe von Padma Lax im Vergleich zu den Dosen als Monopräparat

\begin{tabular}{|c|c|c|c|}
\hline Lateinischer Name & Deutscher Name & $\begin{array}{l}\text { Dosis in Padma Lax, } \\
\text { mg/Tablette }\end{array}$ & $\begin{array}{l}\text { Dosis als } \\
\text { Monopräparat, mg }\end{array}$ \\
\hline Aloe bardadensis bzw. Aloe ferox & $\begin{array}{l}\text { Aloe (entspr. Menge an } \\
\text { Hydroxyanthracenderivaten) }\end{array}$ & 2,5 & $10-40$ \\
\hline Rhamnus purshiana & Cascararinde & 52,5 & $300-1000$ \\
\hline Frangula alnus & Faulbaumrinde & 52,5 & $150-200$ \\
\hline Rheum officinale; Rheum palmatum & Rhabarberwurzel & 70 & $1000-2000$ \\
\hline Marsdenia condurango & Kondurangowurzel & 10 & $3000-4000$ \\
\hline Gentiana lutea & Enzianwurzel & 35 & 1000 \\
\hline Zingiber officinale & Ingwerwurzel & 70 & $1000-2000$ \\
\hline Jateorhiza palmata & Colombowurzel & 10 & KA \\
\hline Strychnos nux-vomica & Brechnusssamen & 1,75 & $\mathrm{KA}$ \\
\hline Piper longum; Piper retrofactum & Langer Pfeffer & 3,5 & KA \\
\hline Terminalia chebula & Myrobalanfrucht & 35 & KA \\
\hline Inula helenium & Alantwurzel & 35 & $\mathrm{KA}$ \\
\hline Natrium sulfuricum & Natriumsulfat (Glaubersalz) & 35 & $10,000-30,000$ \\
\hline Natrium hydrogencarbonicum & Natriumhydrogencarbonat & 15 & KA \\
\hline Kaolinum & Kaolin & 25 & KA \\
\hline
\end{tabular}

$\mathrm{KA}=$ Keine Angaben. im Gegenzug die herrschende Schulmedizin pauschal ablehnen.

\section{Die Beratung anhand eines tibetischen Medikaments}

In einer Apotheke kann einem Kunden während einer Beratung für die Qualität eines Medikaments nicht eine Studie als Argument vorgestellt werden. Kunden - in den allermeisten Fällen medizinisch-wissenschaftliche Laien - verstehen die komplizierte Sprache einer klinischen Studie nicht. Ausserdem finden auch in einer ausgesprochen beratungsintensiven Apotheke weder die Apotheker noch die Kunden so viel Zeit für ein solches $\mathrm{Ge}$ spräch. In der Folge sollen Möglichkeiten aufgezeigt werden, wie die Erkenntnisse über den Multi-TargetWirkungsmechanismus eines pflanzlichen Vielstoffgemischs aus der Tibetischen Medizin in die Beratung über Obstipation integriert werden können (das beschriebene Präparat ist in der Schweiz unter den Markennamen Padma Lax bzw. Padmed Laxan im Handel; Padmed Laxan wird von der Grundversicherung erstattet). Natürlich kennt ein komplementärmedizinisch ausgerichteter Apotheker nicht alle Details der in der Folge vorgestell- ten Studie. Er liest aber regelmässig Fachliteratur und baut darin gefundene, interessante Erkenntnisse in seine Beratung im Alltag ein.

\section{Die Studie}

In dieser Zeitschrift ist kürzlich eine Studie publiziert worden, die MultiTarget-Wirkungsmechanismen eines pflanzlichen Vielstoffgemischs der Tibetischen Medizin bei Obstipation vorstellte [3]. Da der Artikel in der SCHWEIZERISCHEN ZEITSCHRIFT FÜR GANZHEITSMEDIZIN publiziert wurde, soll die Studie hier nur kurz resümiert werden. Als Hintergrund der Studie diente die Tatsache, dass das tibetische Medikament aus 12 gemahlenen Pflanzendrogen und 3 Mineralien besteht und daher mit grosser Wahrscheinlichkeit nicht nur eine laxative Wirkung aufweist. Eine Recherche klinischer Studien bringt dann Erkenntnisse zutage, die auf ein vielschichtiges Wirkmuster hindeuten. So kann mit diesem Präparat bei Verstopfungen verschiedener Genese eine laxative Wirkung mit einem weicheren Stuhl erzielt werden. Darüber hinaus werden aber auch Begleiterscheinungen der Verstopfung wie Blähungen, Schmerzen, Krämpfe sowie Flatulenz behandelt und vermindert, die z.B. zum Erscheinungsbild eines Irritable Bowel Syndrome (IBS), also eines Reizdarms, gehören. Die gute Wirksamkeit und Verträglichkeit kann auf die synergistische Wirkung der 15 Inhaltsstoffe zurückgeführt werden, unter denen bekannte abführende Pflanzendrogen, aber auch solche mit einer karminativen, spasmolytischen und verdauungsfördernden Wirkung zu finden sind. Die Konzentration einzelner Inhaltsstoffe ist zum Teil weit unter der Konzentration, die erforderlich ist, wenn die betreffende Pflanze als Monodroge eingesetzt wird (Tab. 1).

\section{Laxativa-Verkäufe in der Apotheke}

Es gibt viele Muster des Verkaufs und der Beratung von Laxativa in der Apotheke. Es gibt Kunden, welche die Apotheke betreten, ein entsprechendes Präparat verlangen und eine Minute später wieder das Geschäft verlassen. Ähnlich verläuft es mit Kunden, die «etwas» gegen Verstopfung verlangen und das Medikament diskussionslos mitnehmen, das man ihnen vorlegt, und ebenso schnell wieder die Apotheke verlassen wie der Kunde, der gezielt ein Präparat verlangt. In 
beiden Fällen wird kein Wort über die Zusammensetzung des verkauften Präparats oder über den Schweregrad der Verstopfung verloren. Es wird auch nicht darüber gesprochen, ob es sich um eine akute oder eine chronische Verstopfung handelt.

Weitere Kunden kommen in die Apotheke und verlangen wiederum «etwas» gegen Verstopfung. Wenn auf synthetische Medikamente ausgerichtete Apotheker wahrscheinlich ein Medikament auf der Basis von Bisacodyl auswählen und sich komplementärmedizinisch ausgerichtete Apotheker allenfalls von Anfang an für das tibetische Vielstoffgemisch entscheiden und das entsprechende Präparat dem Kunden vorlegen, folgt die eher eigenartige Frage, ob man auch noch etwas anderes habe bzw. ob man auch ein bestimmtes Präparat habe, das er dann nennt. Der Kunde hat ja «etwas» gegen Obstipation verlangt und nicht von Anfang an nach diesem bestimmten Präparat gefragt. Warum spricht er erst im zweiten Moment davon? Für den beratenden Apotheker wird die Situation noch komplizierter, wenn dieser Kunde dann eine Werbung hervorzieht und auf ein Präparat hinweist, das darin empfohlen wird, oder sich auf eine Person bezieht, die ihm das Präparat empfohlen habe, auf das er dann verweist. Mit dem Satz «Ja, natürlich habe ich noch viele andere abführende Präparate und auch dasjenige, das sie nun verlangen» tönt der Apotheker feinfühlig an, dass er etwas ungehalten ist. Ein solches Gespräch kann dann in die Richtung gehen, dass der Kunde misstrauisch wird, gar nichts kauft oder sich doch von der ersten Empfehlung des Apothekers überzeugen lässt oder, was meistens stattfindet, doch das in zweiter Linie erwähnte bzw. verlangte Präparat kauft.

Es kommt auch immer wieder vor, dass Kunden gezielt ein natürliches Abführmittel verlangen. In diesem Fall werden eher schulmedizinisch ausgerichtete Apotheker eher ein Anthrachinon-haltiges Laxativum empfehlen, während Apotheker mit kom- plementärmedizinischem Ansatz wiederum das tibetische Vielstoffgemisch empfehlen, da es eine rein natürliche Zusammensetzung aufweist (Tab. 1).

\section{Obstipationsberatung}

Neben diesen hier vorgestellten, eher kurzen Gesprächen und Verkaufssituationen gibt es in der Apotheke aber auch oft ausführliche Beratungsgespräche über Obstipation, Ursachen, nichtmedikamentöse Behandlungsmöglichkeiten und verschiedenartige Laxantien sowie die Auswahl des im gegebenen Moment geeignetsten Abführmittels. In einem solchen Fall wird meistens über chronische Obstipation gesprochen. Aus bekannten Gründen werden solche Gespräche vor allem mit Frauen geführt. Diese leiden schon seit langer Zeit an Verstopfung, haben schon Verschiedenes dagegen ausprobiert und wissen auch meistens über die nichtmedikamentösen Massnahmen Bescheid. «Ich trinke genug, sicher einen Liter pro Tag» ist ein in der Apotheke oft gehörter Satz. Wenn man dann erwidert, 2-3 Liter seien besser, antwortet die Kundin mit einem Seufzen und sagt, sie habe einfach nicht genügend Durst für solche Mengen. Und das ist wirklich für viele Frauen ein Problem. Der tägliche Durst ist auf einen Liter ausgerichtet, aber sie sollten 2-3 Liter trinken. Wie kann das bewerkstelligt werden? Die ballaststoffreiche Ernährung ist dann wiederum ein Punkt, mit dem die meisten Frauen kein Problem haben. Doch schon der Bewegungsmangel ist wieder ein Aspekt, bei dem Verbesserungspotenzial vorhanden wäre.

\section{Anthrachinon-haltige Laxantien}

Erwähnt der Apotheker in einem solchen Gespräch Laxantien auf der Basis von Senna, Faulbaum oder Chinesischem Rhabarber, dann erinnern sich gewisse Kunden, dass diese Abführmittel früher im Verdacht stan- den, Abhängigkeit zu erzeugen und kanzerogene Eigenschaften zu besitzen. Sogar Fachleute gingen deswegen dazu über, Laxantien auf der Basis von Anthrachinonen nicht mehr zu empfehlen. Doch spätestens seit dem Konsensuspapier von 1999 gibt es keine wissenschaftlich-rationalen Gründe mehr, die gegen die Verwendung solcher Laxantien sprechen [4]. Dies ist auch für unser tibetisches Vielstoffgemisch wichtig, das gleich 4 Pflanzendrogen auf der Basis von Anthrachinonen enthält (Tab. 1).

\section{Nicht nur Verstopfung}

Bei einem ausführlichen Gespräch über Verstopfung wird auch über mögliche krankheitsbedingte Ursachen der vorliegenden Obstipation gesprochen. Manchmal taucht dann eine Reihe weiterer Symptome wie Blähungen, Flatulenz sowie Bauchkrämpfe auf, die auf ein IBS (Reizdarm) schliessen lassen. Und auf einmal geht es bei dem Gespräch nicht mehr nur um Verstopfung, sondern um zusätzliche Beschwerden des Verdauungsapparats, die möglicherweise zu einer Kaskade von Symptomen und Funktionsstörungen führen. In einem solchen Fall kann man das tibetische Vielstoffgemisch ganz besonders empfehlen, da seine Zusammensetzung eben nicht nur für eine bessere Stuhlfrequenz und -konsistenz sorgt, sondern auch zur Verbesserung der Symptome bzw. des IBS führt. Verschiedene Inhaltsstoffe des Gemischs wirken blähungshemmend, spasmolytisch, die Peristaltik anregend usw. (Tab. 2). Durch die Förderung der Magensaftsekretion wird bereits die Peristaltik verbessert, was zu einer Verbesserung des Stuhlgangs beiträgt. Das Vielstoffgemisch wirkt dank der Rhabarberwurzel nicht nur abführend, sondern gleichzeitig auch antidiarrhoisch. Damit hebt es eine häufige, unerwünschte Wirkung herkömmlicher Laxantien auf, denn diese bewirken das Gegenteil einer Verstopfung, nämlich einen Durch- 
Tab. 2. Wirkprofil der Inhaltsstoffe des pflanzlichen Vielstoffgemischs

\begin{tabular}{|c|c|c|c|c|c|c|c|c|c|c|c|c|c|}
\hline Deutscher Name & Laxativ & $\begin{array}{l}\text { Peristal- } \\
\text { tik anre- } \\
\text { gend }\end{array}$ & $\begin{array}{l}\text { Spas- } \\
\text { mo- } \\
\text { lytisch }\end{array}$ & $\begin{array}{l}\text { Kar- } \\
\text { mi- } \\
\text { nativ }\end{array}$ & $\begin{array}{l}\text { Magen- } \\
\text { saft- } \\
\text { sekretion }\end{array}$ & $\begin{array}{l}\text { Bitter- } \\
\text { stoffe }\end{array}$ & $\begin{array}{l}\text { Gerb- } \\
\text { stoffe }\end{array}$ & $\begin{array}{l}\text { Ad- } \\
\text { sorptiv }\end{array}$ & $\begin{array}{l}\text { Hyd- } \\
\text { ra- } \\
\text { gog }\end{array}$ & $\begin{array}{l}\text { Desensi- } \\
\text { bilisie- } \\
\text { rend }\end{array}$ & $\begin{array}{l}\text { Antiab- } \\
\text { sorptiv }\end{array}$ & $\begin{array}{l}\text { Anti } \\
\text { diarrho- } \\
\text { isch }\end{array}$ & $\begin{array}{l}\text { Säure- } \\
\text { bin- } \\
\text { dend }\end{array}$ \\
\hline Aloe & $\mathrm{x}$ & $\mathrm{x}$ & & & & $\mathrm{x}$ & & & $\mathrm{x}$ & & & & \\
\hline Cascararinde & $\mathrm{x}$ & & & & & & & & $\mathrm{x}$ & & $\mathrm{x}$ & & \\
\hline Faulbaumrinde & $\mathrm{x}$ & & & & & & & & $\mathrm{x}$ & & $\mathrm{x}$ & & \\
\hline Rhabarberwurzel & $\mathrm{x}$ & & & & & & $\mathrm{x}$ & & & & & $\mathrm{x}$ & \\
\hline Kondurangowurzel & & & & & $\mathrm{x}$ & $\mathrm{x}$ & & & & & & & \\
\hline Enzianwurzel & & $\mathrm{x}$ & & & $\mathrm{x}$ & $\mathrm{x}$ & & & & & & & \\
\hline Ingwerwurzel & & $\mathrm{x}$ & $\mathrm{x}$ & $\mathrm{x}$ & & & & & & & & & \\
\hline Colombowurzel & & & & & & $\mathrm{x}$ & & & & & & $\mathrm{x}$ & \\
\hline Brechnusssamen & & & & & & $\mathrm{x}$ & & & & & & & \\
\hline Langer Pfeffer & & $\mathrm{x}$ & & & & & & & & $\mathrm{x}$ & & & \\
\hline Myrobalanfrucht & & & & & & $\mathrm{x}$ & $\mathrm{x}$ & & & & & & \\
\hline Alantwurzel & & & & & $\mathrm{x}$ & $\mathrm{x}$ & & & & & & & \\
\hline $\begin{array}{l}\text { Natriumsulfat } \\
\qquad \text { (Glaubersalz) }\end{array}$ & $\mathrm{x}$ & & & & & & & & & & & & \\
\hline $\mathrm{NaHCO}_{3}$ & & & & & & & & & & & & & $\mathrm{x}$ \\
\hline Kaolin & & & & & & & & $\mathrm{x}$ & & & & & \\
\hline
\end{tabular}

fall. Die Wirksamkeit und Sicherheit des Präparats bei Verstopfung und weiteren Beschwerden des Verdauungsapparats wurde mehrfach dokumentiert [5, 6]. Dank des Synergismus der verschiedenen Inhaltsstoffe sind diese im vorliegenden Präparat zum Teil viel niedriger dosiert als dies in abführenden Präparaten erforderlich ist, die nur einen Wirkstoff enthalten (Tab. 1). Dies wirkt sich wiederum positiv auf die Verträglichkeit aus.

\section{Verwendung}

Dank seiner Zusammensetzung kann das tibetische Medikament bei einer herkömmlichen Verstopfung empfohlen werden. Aufgrund seiner speziellen Zusammensetzung besteht kaum die Möglichkeit, dass eine überschiessende Wirkung eintritt, d.h., dass der Darm der betroffenen Person mit einer Diarrhö reagiert. Wenn im Verlauf des Beratungsgesprächs weitere Symptome wie Krämpfe, Flatulenz usw. zum Vorschein kommen, dann eignet sich das Präparat dank der Inhaltsstoffe, die gegen diese Symptome wirken, ganz besonders. Es kann aber auch der Fall sein, dass jemand in einer Apotheke eine Beratung über diese Beschwerden in Anspruch nimmt und nicht in erster Linie von Verstopfung spricht. Diese wird dann erst im Verlauf des Gesprächs erwähnt. Doch auch hier kann das tibetische Präparat bestens empfohlen werden, da es - wie erwähnt - nicht nur laxativ, sondern auch spasmolytisch und karminativ wirkt. Die Verstopfung und deren Begleitsymptome hängen miteinander zusammen und sollten ganzheitlich behandelt werden. Diese Voraussetzung erfüllt das pflanzliche Vielstoffgemisch aus der Tibetischen Medizin.

\section{Fazit}

Der Verkauf eines Laxativums in einer Apotheke kann - aufgrund des Verhaltens des Kunden oder (leider) wegen der Struktur der Apotheke sehr kurz und ohne Beratung über die Bühne geben. Engagierte Apothekerinnen und Apotheker versuchen aber jeweils, mit der Kundschaft ins Gespräch zu kommen, um allfällige Hintergründe herauszufinden und dann ein geeignetes Laxativum empfehlen zu können, das im Zusammenhang mit nichtmedikamentösen Massnahmen angewendet werden kann.

Apothekerinnen und Apotheker mit einer komplementärmedizinischen Ausrichtung empfehlen oft ein tibetisches Vielstoffgemisch, dessen Wirksamkeit und Sicherheit bei Verstopfung und anderen Beschwerden des Verdauungsapparats mehrfach dokumentiert wurde. Das Präparat erfüllt zudem alle schulmedizinischen Kriterien und kann daher auch von ausschliesslich auf die Schulmedizin ausgerichteten Apothekerinnen und Apothekern empfohlen werden. Dank seiner speziellen Zusammensetzung eignet es sich zur Behandlung einfacher Verstopfungen, aber auch von Verstopfungen im Zusammenhang mit anderen Beschwerden wie IBS.

\section{Literatur}

1 www.interpharma.ch.

2 www.pharmaSuisse.ch

$>3$ Simitz S, Vennos C: Multi-Target-Wirkungsmechanismen des pflanzlichen Vielstoffgemischs Padma Lax bei Obstipation. Schweiz Z Ganzheitsmed 2015;27:347-353.

4 Hitzenberger G, et al: Expertenforum: Obstipation und Laxantien. Praxis 1999;88:1269-1274.

$>5$ Uehleke B, et al: Die Tibetische Rezeptur Padma Lax in der ärztlichen Praxis: Eine retrospektive Fallstudie bei Obstipation mit 174 Patienten. Forsch Komplementmed 2013;20 (suppl 2):8-13.

6 Sallon S, et al: A novel treatment for constipation-predominant irritable bowel syndrome using Padma Lax, a Tibetan herbal formula. Digestion 2002;65:161-171. 